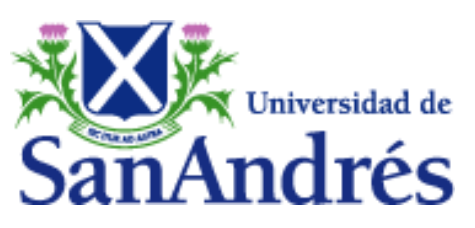

"Social Security as Markov Equilibrium in OLG Models: A Note"

Por Martín Gonzalez Eiras (Universidad de San Andrés \& CONICET).

D.T.: $\mathbf{N}^{0} 105$

Septiembre, 2010

Vito Dumas 284, B1644BID, Victoria, San Fernando, Buenos Aires, Argentina Teléfono 4725-7053, Fax 4725-7010

Email,economia@udesa.edu.ar 


\title{
Social security as Markov equilibrium in OLG models: a note*
}

\author{
Martín Gonzalez-Eiras \\ Universidad de San Andrés; CONICET ${ }^{\dagger}$
}

July 14, 2010

\begin{abstract}
I refine and extend the Markov perfect equilibrium of the social security policy game in Forni (2005) for the special case of logarithmic utility. Under the restriction that the policy function be continuous, instead of differentiable, the equilibrium is globally well defined and its dynamics always stable.
\end{abstract}

Keywords: Social security; Overlapping generations models; Markov equilibria

Forni (2005) analyzes an economy with two-period lived overlapping generations, capital accumulation, exogenous labor supply, and a proportional wage tax whose receipts fund a lump-sum transfer to retirees, i.e. a PAYGO system. He argues that in a median voter environment without commitment and trigger strategies, there exist equilibria in which political decision makers support strictly positive social security taxes because they expect future social security benefits to be a decreasing function of the capital stock. ${ }^{1}$ Under the restriction that the policy function be differentiable, the author characterizes equilibria of this type supporting a strictly positive tax rate $\tau_{t}$ as long as the capital stock, $k_{t}$, moves inside an interval $[\underline{\mathrm{k}}, \bar{k}]$ that depends on parameter values and satisfies $\underline{\mathrm{k}}>0$ and $\bar{k}<\infty$.

The objective of this note is to refine and extend the results in Forni (2005) that correspond to the special case of logarithmic utility and Cobb-Douglas production function. ${ }^{2}$ First, I derive conditions for a stable steady state to exist, requiring stronger restrictions on parameters. Second, for some $k_{0} \in[\underline{\mathrm{k}}, \bar{k}]$ the social security system collapses in finite time, and therefore the proposed policy function cannot be a solution for the entire interval $[\underline{\mathrm{k}}, \bar{k}]$. Finally, requiring that the policy function be continuous, but not necessarily differentiable, I extend the domain of the policy function to $k_{t} \in[0, \infty)$.

The two key dynamic equations in Forni (2005) are:

$$
\begin{aligned}
\tau^{F}(k) & =\frac{\alpha}{1-\alpha}\left(C k^{-\frac{1+\alpha \beta}{1+\beta}}-1\right), \\
g\left(k_{t+1}\right) \equiv \beta k_{t+1}+C k_{t+1}^{1-\theta} & =\frac{\beta}{1+n}\left(k_{t}^{\alpha}-\alpha C k_{t}^{\alpha-\theta}\right) \equiv h\left(k_{t}\right) .
\end{aligned}
$$

${ }^{*}$ I thank Enrique Kawamura, Dirk Niepelt, and an anonymous referee for comments, and Lorenzo Forni for helpful discussions. All errors are mine.

${ }^{\dagger}$ Vito Dumas 284, B1644BID Victoria, Pcia. Buenos Aires, Argentina. E-mail: mge@alum.mit.edu.

${ }^{1}$ Further work on Markov perfect equilibrium in economies with social security include Chen and Song (2009), Gonzalez-Eiras and Niepelt (2008), and Mateos-Planas (2008).

${ }^{2}$ Forni (2005) also solves the model numerically for the case of general CRRA preferences. 
The first equation gives the equilibrium policy function under the restriction that this be a differentiable function of $k$, with $\beta$ being the time discount factor, $\alpha$ the share of capital in production, and $C \geq 0$ a constant of integration. The second equation, in which $n$ is the net rate of population growth and $\theta \equiv(1+\beta \alpha) /(1+\beta)$, results after substituting the policy function $\tau^{F}$ into the capital accumulation expression

$$
(1+n) k_{t+1}=\frac{1-\alpha}{1+\beta}\left[\beta\left(1-\tau_{t}\right) k_{t}^{\alpha}-\frac{\tau_{t+1} k_{t+1}(1+n)}{\alpha}\right] .
$$

The restriction that $\tau_{t} \in(0,1)$ leads to the constraint that (1) and (2) only hold for $k_{t} \in\left(\underline{\mathrm{k}}=(\alpha C)^{1 / \theta}, \bar{k}=C^{1 / \theta}\right)$, where $\tau^{F}(\underline{\mathrm{k}})=1$ and $\tau^{F}(\bar{k})=0$. Two properties of (2) that I will repeatedly use are that the relation between $k_{t}$ and $k_{t+1}$ is continuous even if it has no closed form solution, and that $g^{\prime}(\cdot)$ and $h^{\prime}(\cdot)$ are continuous functions. Furthermore, the relation between $k_{t}$ and $k_{t+1}$ is continuous at $\bar{k}$ since taxes become zero at this point. Finally, since $\lim _{k \rightarrow \underline{\mathrm{k}}} g(k)>0, \lim _{k \rightarrow \underline{\mathrm{k}}} h(k)=0, \lim _{k \rightarrow \infty} g^{\prime}(k)=\beta>0$, and $\lim _{k \rightarrow \infty} h^{\prime}(k)=0$, if there exists a stable steady state, $k_{s}^{s s}$, there also exists an unstable steady state $k_{u}^{s s}$, with $\underline{\mathrm{k}}<k_{u}^{s s}<k_{s}^{s s} .{ }^{3} 4$

I start by deriving conditions for a stable steady state to exist. When $C=0$ the dynamics of capital reduce to the case of an economy without taxes which has a unique stable steady state. Increases in $C$ shift the function $g(k)$ upwards and $h(k)$ downwards. Thus, there exists a $\bar{C}$ such that for $C>\bar{C}$ the economy has no non-trivial steady state. There is no closed form expression for $\bar{C}$ but it can be characterized by ${ }^{5}$

$$
\bar{C}=\inf \{C \text { s.t. } g(k) \leq h(k) \quad \forall \quad k\} .
$$

The restriction $C \leq \bar{C}$ is not sufficient to guarantee that at a stable steady state a positive social security tax rate is sustained. For this we also need that $k_{s}^{s s}<\bar{k}$. Otherwise the economy would accumulate, in finite time, a level of capital $k_{t}>\bar{k}$ for which there are no intergenerational transfers. By backward induction, no positive tax rates would be supported during the transition. If a steady state exists, a necessary and sufficient condition for $k_{s}^{s s}<\bar{k}$ is

$$
g(\bar{k}) \geq h(\bar{k}) .
$$

To prove this claim, suppose to the contrary that $g(\bar{k})<h(\bar{k})$. This implies that when $k_{t}=\bar{k}$ then $k_{t+1}>k_{t}$, and therefore $\tau_{t}=0$. The dynamics in (3) with no taxes show that the capital stock would continue to increase until a steady state is reached, and thus $k_{s}^{s s}>\bar{k}$. Conversely, if a steady state exists and (4) holds, then since locally around $\bar{k}$ $k_{t+1}<k_{t}$, it cannot be that $k^{s s}>\bar{k}$. This follows since for $k>\bar{k}$ the dynamics corresponds

\footnotetext{
${ }^{3}$ This follows from the fact that a stable steady state is characterized by $h\left(k_{s}^{s s}\right)=g\left(k_{s}^{s s}\right)$ and $h^{\prime}\left(k_{s}^{s s}\right)<$ $g^{\prime}\left(k_{s}^{s s}\right)$, and that $\lim _{k \rightarrow \underline{\mathrm{k}}} h(k)<\lim _{k \rightarrow \underline{\mathrm{k}}} g(k)$. By continuity of $(2)$, and of $h^{\prime}(\cdot)$ and $g^{\prime}(\cdot)$, then there must exist a $k \in\left(\underline{\mathrm{k}}, k_{s}^{s s}\right)$ that satisfies $h(k) \stackrel{=}{=} g(k)$ and $h^{\prime}(k)>g^{\prime}(k)$, i.e. $k$ is an unstable steady state.

${ }^{4}$ To rule out the existence of more than two steady states, a sufficient condition would be that $h^{\prime \prime}(k)<$ $g^{\prime \prime}(k)<0 \quad \forall k \geq \underline{\mathrm{k}}$. If there are more steady states in general there are an even number of them. I will assume that at most two steady states exist. This assumption does not affect the results derived.

${ }^{5}$ Alternatively, under the assumption that at most two steady states exist, $\bar{C}$ can be characterized by the following system of two equations in the two unknowns, $k$, and $\bar{C}$,

$$
\begin{aligned}
g(k) & =h(k), \\
g^{\prime}(k)=\beta+\bar{C}(1-\theta) k^{-\theta} & =\frac{\beta}{1+n}\left(\alpha k^{\alpha-1}+\alpha(\theta-\alpha) \bar{C} k^{\alpha-\theta-1}\right)=h^{\prime}(k) .
\end{aligned}
$$

An upper bound on $\bar{C}, \overline{\bar{C}}$, can be found when $h^{\prime \prime}(k)<g^{\prime \prime}(k)<0 \forall k \geq \underline{\mathrm{k}}$. This is done by solving for $g^{\prime}(\underline{\mathrm{k}})=h^{\prime}(\underline{\mathrm{k}})$, and results in $\overline{\bar{C}}=\frac{1}{\alpha}\left[\frac{\beta^{2}(1+\alpha \beta)^{2}}{(1+n) \alpha(1+\beta)}\right]^{\frac{\theta}{1-\alpha}}$.
} 
to an economy without taxes, and thus $k_{t+1}<k_{t}$ holds $\forall k_{t}>\bar{k}$. Replacing $\bar{k}=C^{\frac{1}{\theta}}$ in (4) results in the following lower bound on $C$

$$
C \geq \underline{\mathrm{C}} \equiv\left(\frac{\beta(1-\alpha)}{(1+n)(1+\beta)}\right)^{\frac{\theta}{1-\alpha}}
$$

Additionally, we need $\underline{\mathrm{C}} \leq \bar{C}$, otherwise a stable steady state that supports a positive social security tax does not exist. These restrictions put bounds on the parameter $C$ for which Forni's proposed policy function, (1), is indeed an equilibrium.

I now show that the lower end of the proposed domain, $\underline{\mathrm{k}}=(\alpha C)^{1 / \theta}$, is not correct. This value satisfies $h(\underline{\mathrm{k}})=0$, and thus $k_{t+1}=0$ from (2). Since $\underline{\mathrm{k}}>0$ (and therefore $0=k_{t+1}<k_{t}=\underline{\mathrm{k}}$ ) this means, by continuity of (2), that in an interval around $\underline{\mathrm{k}}$ the capital stock is decreasing in time $\left(k_{t+1}<k_{t}\right)$. From before, if a stable steady state exists then, by continuity of (2), and of $h^{\prime}(\cdot)$ and $g^{\prime}(\cdot)$, there must exist an unstable steady state and $\underline{\mathrm{k}}<k_{u}^{s s}<k_{s}^{s s}$. Thus $k_{t+1}<k_{t}$ when $k_{t} \in\left[\underline{\mathrm{k}}, k_{u}^{s s}\right)$. Therefore if $k_{0} \in\left[\underline{\mathrm{k}}, k_{u}^{s s}\right)$ then, in finite time, $k_{t}<\underline{\mathrm{k}}$ and the social security system would collapse. By backward induction there cannot be a positive tax rate along these paths. ${ }^{6}$

We can therefore restate the results in Forni (2005) by saying that as long as $\underline{\mathrm{C}} \leq \bar{C}$ there exist multiple expectational equilibria that sustain a PAYGO system, indexed by $C \in[\underline{\mathrm{C}}, \bar{C}]$. His proposed solution for the policy function, (1), holds as long as the initial capital, $k_{0}$ satisfies $k_{0} \in\left[k_{u}^{s s}, \bar{k}\right] .^{7}$

I now lift the assumption that the policy function has to be differentiable in order to extend the domain of the equilibrium policy function to $k_{t} \in[0, \infty)$. Before doing this I will provide a formal definition of the Markov perfect equilibrium adopted in this note. ${ }^{8}$ The median voter is restricted to play Markovian strategies, i.e. strategies that prescribe a social security tax rate that can only depend on the current pay-off relevant state, the capital stock, $\tau_{t}=\tau\left(k_{t}\right)$. Denoting by $\Psi\left(k_{t}, \tau_{t}, \tilde{\tau}\left(k_{t+1}\right)\right)$ the indirect utility function of the median voter in period $t$ (which depends on the current tax, $\tau_{t}$, and on the expectation of how future taxes are affected by the state in the following period, $\left.\tilde{\tau}\left(k_{t+1}\right)\right)$, the program that characterizes the policy choice of the median voter is,

$$
\begin{aligned}
\max _{0 \leq \tau_{t} \leq 1} \Psi\left(k_{t}, \tau_{t}, \tilde{\tau}\left(k_{t+1}\right)\right) & \\
\text { s.t. } k_{t+1} & =\frac{1-\alpha}{1+\beta}\left[\frac{\beta\left(1-\tau_{t}\right) k_{t}^{\alpha}}{1+n}-\frac{\tilde{\tau}\left(k_{t+1}\right) k_{t+1}}{\alpha}\right], \\
\tilde{\tau}(\cdot) & \in \mathcal{C}^{0}
\end{aligned}
$$

where the first constraint in the maximization problem is the capital accumulation expression (3) with future taxes given by $\tilde{\tau}\left(k_{t+1}\right)$, and the second restriction requires the expected policy function to be continuous. In a rational expectations equilibrium, the anticipated policy function coincides with the optimal one, i.e. the Markov perfect equilibrium of the policy game is given by,

$$
\tau\left(k_{t}\right)=\arg \max _{0 \leq \tau_{t} \leq 1} \Psi\left(k_{t}, \tau_{t}, \tau\left(k_{t+1}\right)\right) \quad \forall k_{t} \geq 0 .
$$

\footnotetext{
${ }^{6}$ Forni $(2005)$ arrives to $(1)$ and $(2)$ under the restriction that current taxes are interior, $\tau_{t} \in(0,1)$ (see his Appendix A), while the correct formulation requires that this condition be satisfied by all future taxes as well (Forni acknowledges this when describing equilibrium dynamics).

${ }^{7}$ Since there are multiple equilibria, one should write $k_{u}^{s s}(C)$ and $\bar{k}(C)$. For simplicity the indexation on $C$ is dropped.

${ }^{8}$ For reference and comparison, see the definition of the MPE in Forni (2005) page 183.
} 
Consider first the case $k<k_{u}^{s s}$. For $k_{t}$ close enough to $k_{u}^{s s}$ the chosen tax would be positive. We can prove this by contradiction. If taxes were zero then $k_{t+1}>k_{u}^{s s}$, which means that $\tau_{t+1}$ is a decreasing function of savings. But this gives an incentive to the current median voter to choose a positive tax rate. ${ }^{9}$ Conjecture that the optimal choice is the tax rate that makes $k_{t+1}=k_{u}^{s s 10}$

$$
\tau^{e}\left(k_{t}\right)=1-\left(\frac{1+\beta}{1-\alpha}+\frac{\tau^{F}\left(k_{u}^{s s}\right)}{\alpha}\right) \frac{(1+n) k_{u}^{s s}}{\beta k_{t}^{\alpha}},
$$

where $\tau^{e}$ is the "extended" policy function. Note that $\tau^{e^{\prime}}>0$, and that by continuity of the dynamics in (3) we have that $\tau^{e}\left(k_{u}^{s s}\right)=\tau^{F}\left(k_{u}^{s s}\right)$. Thus, the policy function is continuous, but not differentiable, at $k_{u}^{s s}$. Equation (5) gives the policy function for $k<k_{u}^{s s}$, as long as the solution is non-negative. Let's call $k_{L}$ the value of the capital stock for which $\tau^{e}\left(k_{L}\right)=0$. For $k<k_{L}$ the chosen tax rate is zero. If $k_{t}<k_{L}$ is close to $k_{L}$, then $\tau_{t}>0$ would imply $k_{t+1}<k_{u}^{s s}$, and even if $\tau_{t+1}>0$ the policy function is an increasing function of the capital stock and the median voter has no incentives to tax. ${ }^{11}$ Again the policy function is continuous at $k_{L}$. For $k_{t}<k_{L}$ and close to the origin, since $k_{t+1}<k_{L}$ and therefore $\tau_{t+1}=0$, we get that $\tau_{t}=0$ as well. Moreover, the dynamics of the capital stock under $\tau^{e}$ transform $k_{u}^{s s}$ into a semi-stable steady state: If $k_{t} \neq k_{u}^{s s}$ is in a neighborhood of $k_{u}^{s s}$, then $k_{t}<k_{t+1}=k_{u}^{s s}$, or $k_{u}^{s s}<k_{t}<k_{t+1}$. Therefore, continuous Markov strategies do not produce the unstable dynamics that Forni (2005) found for the simple logarithmic utility and Cobb-Douglas production functions model.

To verify the conjecture we must rule out other solutions for $k_{t} \in\left[k_{L}, k_{u}^{s s}\right)$. First consider the case that the median voter deviates to $\tau_{t}>\tau^{e}\left(k_{t}\right)$. By construction of $\tau^{e}$, $k_{t+1}<k_{u}^{s s}$. Thus, the tax rate for $t+1$ would be an increasing function of the capital stock, and therefore the median voter would have no incentive to tax at time $t$. Conversely, she would never choose a positive tax rate below $\tau^{e}\left(k_{t}\right)$ since the marginal benefit of increasing taxes is positive (remember that the unconstrained choice would be $\tau^{F}\left(k_{t}\right)>\tau^{e}\left(k_{t}\right)$ ).

To complete the characterization of the equilibrium policy function I will show that $\tau^{e}(k)=0$ for $k>\bar{k}$. According to (1), when $k_{t}=\bar{k}$ the median voter finds optimal to set a tax of zero, even if $\tau_{t+1}$ (given also by (1) since $k_{s}^{s s}<k_{t+1}<\bar{k}$ ) is positive and decreasing in the capital stock. Thus, the incentive of an increase in future benefits in exchange of a reduction in current savings is not strong enough to induce the median voter to tax her constituency. For values of $k_{t}>\bar{k}$, this incentive is even smaller $\left(k_{t+1}\left(k_{t}\right)\right.$ is an increasing function, and $-\tau^{F^{\prime}}$ is a decreasing function of $k$ ). In fact, (1) would call for negative taxes. Since this is not possible, the chosen tax has to be zero. Thus $\tau^{e}(k)=0$ for $k>\bar{k}$, and the policy function is once more continuous at $\bar{k}$. Figure 1 represents qualitatively the policy function, $\tau^{e}$, and capital accumulation under it (in the figure $k_{H}$ stands for $\bar{k}$ ).

Summarizing, I have shown that assuming that the policy function has to be continuous, but not necessarily differentiable, results in an equilibrium that is valid globally for the economy studied in Forni (2005). Multiple expectational equilibria exist for values

\footnotetext{
${ }^{9}$ This reasoning requires that the dynamics without taxes in (3) applied to $k_{u}^{s s}$ result in $k_{t+1}<\bar{k}$. Otherwise repeat the logic from an initial $k_{t}$ low enough such that $k_{t+1}<\bar{k}$. Although this indicates that other expectational equilibria might exist, the policy function derived would still be the unique continuous one.

${ }^{10}$ This relation comes from $(3)$ when $k_{t+1}=k_{u}^{s s}$, and expected social security benefits are given by $\tau^{F}\left(k_{u}^{s s}\right)$.

${ }^{11}$ The reason for having an upward sloping tax schedule, which counters the logic in Forni (2005), is that as $k_{t}$ evolves from a low initial level it will "hit" the domain of the upward sloping section of the policy function, $\left[k_{L}, k_{u}^{s s}\right)$, only once. Generically it would be the case that $k_{t}<k_{L}<k_{t+1}<k_{t+2}=k_{u}^{s s}$. See Figure 1, where $k_{H}$ stands for $\bar{k}$.
} 
of $C \in[\underline{\mathrm{C}}, \bar{C}]$. If there is an equilibrium with positive intergenerational transfers, the policy function is initially zero, later rises up to a maximum corresponding to $k=k_{u}^{s s}$, then decreases up to $\bar{k}$, being zero thereafter. Thus, an economy that starts with a low level of capital will evolve to a steady state with a (relatively) high PAYGO tax rate and low capital intensity. Since this steady state is semi-stable, a perturbation can send the economy to another steady state with a lower PAYGO tax rate and higher capital intensity. The refinements made in this note, and the richer description of the dynamics, would also be useful to other politico-economic models that build on Forni (2005). ${ }^{12}$

\section{References}

Chen, K., Song, Z., 2009. Markovian Social Security in Unequal Societies. University of Oslo working paper.

Forni, L., 2005. Social security as Markov Equilibrium in OLG models. Review of Economic Dynamics 8 (1), 178-194.

Gonzalez-Eiras, M., Niepelt, D., 2008. The future of social security. Journal of Monetary Economics 55 (2), 197-218.

Mateos-Planas, X., 2008. A quantitative theory of social security without commitment. Journal of Public Economics 92 (3-4), 652-671.

Sand, E., Razin, A., 2007. The Political-Economy Positive Role of the Social Security System in Sustaining Immigration (But not Vice Versa). Tel-Aviv University working paper.

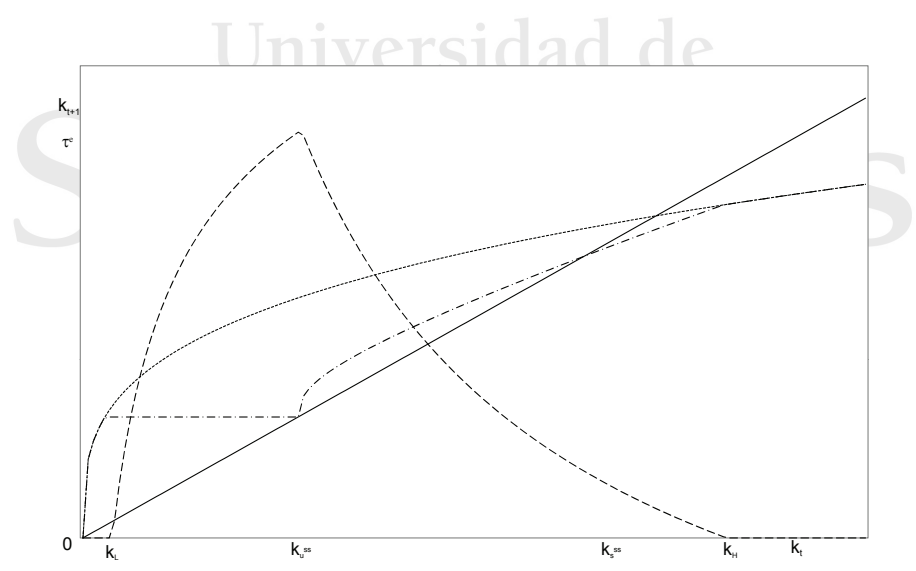

Figure 1: Policy function and capital accumulation. $\tau^{e}(---)$, capital accumulation under $\tau^{e}(-\cdots)$, capital accumulation without taxes $(\cdots), 45^{\circ}$ line $(-)$. Here $k_{H}$ stands for $\bar{k}$.

\footnotetext{
${ }^{12}$ For example, Sand and Razin (2007) study the interaction of social security and immigration policies in an OLG economy in which the young median voter chooses the social security tax rate and the migration quota.
} 